\title{
APPROXIMATE SOLUTION OF FRACTIONAL INTEGRO-DIFFERENTIAL EQUATIONS BY LEAST SQUARES METHOD
}

\author{
D. JABARI SABEG ${ }^{1, *}$, R. EZZATI ${ }^{2}$ AND K. MALEKNEJAD ${ }^{2}$ \\ ${ }^{1}$ Department of Mathematics, Bonab Branch, Islamic Azad University, Bonab, Iran \\ ${ }^{2}$ Department of Mathematics, Karaj Branch, Islamic Azad University, Karaj, Iran \\ *Corresponding author: davood.jabari@bonabiau.ac.ir
}

\begin{abstract}
In this paper, least squares approximation method is developed for solving a class of linear fractional integro-differential equations comprising Volterra and Fredhlom cases. This method is based on a polynomial of degree $n$ to compute an approximate solution of these equations. The convergence analysis of the proposed method is proved. In addition, to show the accuracy and the efficiency of the proposed method, some examples are presented.
\end{abstract}

\section{INTRODUCTION}

Fractional calculus is a significant branch of mathematics that is used in many fields of science and engineering [2-4]. Many researchers have investigated the analytic results on the existence and uniqueness of solutions of the fractional differential equations [5-8]. As we know, for most fractional differential equations, there are not method to obtain analytic solutions, so numerical techniques must be used. During the past years, methods for solving fractional differential equations are developed. Additionally, some methods have recently been emerged, such as the Adomian decomposition method [10,11], the operational matrix [12,13], the collocation method $[14,16]$, etc.

Received 2018-10-06; accepted 2018-11-27; published 2019-03-01.

2010 Mathematics Subject Classification. 26A33, 45A05.

Key words and phrases. fractional differential equation; least squares; approximation method; convergence analysis.

(C)2019 Authors retain the copyrights of their papers, and all open access articles are distributed under the terms of the Creative Commons Attribution License. 
In this paper, by using least squares approximation, a numerical method has been shown to linear fractional integro-differential equations in the following form

$$
D^{\alpha} y(t)=p(t) y(t)+f(t)+\lambda_{1} \int_{0}^{1} k_{1}(t, x) y(x) d x+\lambda_{2} \int_{0}^{t} k_{2}(t, x) y(x) d x \quad t \in I=[0,1]
$$

with the initial conditions

$$
y^{(k)}(0)=d_{k} i=0, . ., m-1, m-1<\alpha \leq m,
$$

where $y^{k}(t)$ stands for the $k$ th-order derivative of $y(t)$ and $D^{\alpha}$ denotes the Riemann-Liouville fractional derivative of order $\alpha$. Clearly, when $\lambda_{1}=0, \lambda_{2}=0$, the above equation reduces to a linear fractional differential equation.

The rest of the paper is organized as follows: In section 2, we will briefly review some notations and definitions of the fractional calculus theory are used in the paper. In Section 3, we introduce the least squares approximation method for solving Eq. (1.1), and discuss its convergence. In Section 4, we show the efficiency of the proposed method with some numerical examples. Section 5 , as the final section, presents a conclusion.

\section{Brief ReView of Fractional Calculus}

In this section, notations and definitions of the fractional calculus theory, which are going to be used in this paper, are presented [1].

2.1. Definition. The Riemann-Liouville fractional integral operator $I^{\alpha}$ of order $\alpha \geq 0$ of a function $f(x)$, is defined as

$$
I^{\alpha} f(x)=\frac{1}{\Gamma(\alpha)} \int_{0}^{x}(x-t)^{(\alpha-1)} f(t) d t, \alpha>0,
$$

where $x>0$ and $\Gamma($.$) is the Euler gamma function.$

The Riemann-Liouville fractional derivative of order $\alpha$ will be denoted by $D^{\alpha}$ and defined by

$$
D^{\alpha} f(x)=\frac{d^{m}}{d x^{m}}\left(I^{m-\alpha} f(x)\right)
$$

where $m-1<\alpha \leq m, m \in \mathbb{N}$ and $m$ is the smallest integer order greater than $\alpha$. We just mention the following property

$$
D^{\alpha} x^{\beta}=\frac{\Gamma(\beta+1)}{\Gamma(\beta+1-\alpha)} x^{\beta-\alpha}, \beta>-1
$$




\section{Method Of SOlution}

In this section, we apply the least squares approximation method for solving Eq. (1.1). We define the following operator

$$
T(t, y(t))=D^{\alpha} y(t)-p(t) y(t)-f(t)-\lambda_{1} \int_{0}^{1} k_{1}(t, x) y(x) d x-\lambda_{2} \int_{0}^{t} k_{2}(t, x) y(x) d x .
$$

We construct Taylor-series expansion for the solution $y(t)$ in Eq.(1.1) as

$$
y(t) \simeq y_{n}(t)=\sum_{r=0}^{n} \frac{y^{(r)}(0)}{r !} t^{r}=\sum_{r=0}^{n} \frac{d_{r}}{r !} t^{r} .
$$

Substituting (3.2) into ( 3.1), we have

$$
\begin{aligned}
T\left(t, y_{n}(t)\right)= & D^{\alpha} y_{n}(t)-p(t) y_{n}(t)-f(t)-\lambda_{1} \int_{0}^{1} k_{1}(t, x) y_{n}(x) d x-\lambda_{2} \int_{0}^{t} k_{2}(t, x) y_{n}(x) d x \\
= & D^{\alpha}\left(\sum_{r=0}^{n} \frac{d_{r}}{r !} t^{r}\right)-p(t) \sum_{r=0}^{n} \frac{d_{r}}{r !} t^{r}-f(t)-\sum_{r=0}^{n} \frac{d_{r}}{r !} \lambda_{1} \int_{0}^{1} k_{1}(t, x) x^{r} d x- \\
& \quad \sum_{r=0}^{n} \frac{d_{r}}{r !} \lambda_{2} \int_{0}^{t} k_{2}(t, x) x^{r} d x \\
= & \sum_{r=0}^{n} \frac{d_{r} \Gamma(r+1)}{r ! \Gamma(r-\alpha+1)} t^{r-\alpha}-p(t) \sum_{r=0}^{n} \frac{d_{r}}{r !} t^{r}-f(t)-\sum_{r=0}^{n} \frac{d_{r}}{r !} \lambda_{1} \int_{0}^{1} k_{1}(t, x) x^{r} d x- \\
& \quad \sum_{r=0}^{n} \frac{d_{r}}{r !} \lambda_{2} \int_{0}^{t} k_{2}(t, x) x^{r} d x \\
= & \sum_{r=0}^{n} d_{r} \gamma_{r}(t)-f(t),
\end{aligned}
$$

where

$$
\gamma_{r}(t)=\frac{\Gamma(r+1)}{r ! \Gamma(r-\alpha+1)} t^{r-\alpha}-p(t) \frac{t^{r}}{r !}-\frac{\lambda_{1}}{r !} \int_{0}^{1} k_{1}(t, x) x^{r} d x-\frac{\lambda_{2}}{r !} \int_{0}^{t} k_{2}(t, x) x^{r} d x
$$

Let $R_{n}(t):=T\left(t, y_{n}(t)\right)-T(t, y(t)), t \in[0,1]$.

Remark 3.1. If $R_{n}(t)=0$, then $y(t)=y_{n}(t)$; if $\lim _{n \rightarrow \infty} R_{n}(t)=0$, then $\lim _{n \rightarrow \infty} y_{n}(t)=y(t)$.

Remark 3.2. For any $t \in[0,1]$, if $R_{n}(t) \equiv 0$, then $y_{n}(t)$ is an exact solution of Eqs. (1.1) and (1.2); if $\lim _{n \rightarrow \infty} R_{n}(t)=0$, then $y_{n}(t)$ converges to the exact solution of Eqs. (1.1) and (1.2).

Let

$$
J=J\left(d_{m}, d_{m+1}, \ldots, d_{n}\right)=\int_{0}^{1} T^{2}\left(t, y_{n}(t)\right) d t
$$

The problem is to find real constants $d_{m}, d_{m+1}, \ldots, d_{n}$ such that these constants will minimize $J$. A necessary condition for the constants $d_{m}, d_{m+1}, \ldots, d_{n}$ to minimize $J$ is that 


$$
\frac{\partial J}{\partial d_{j}}=0
$$

for each $j=m, m+1, \ldots, n$. By referring (3.3), we get

$$
\frac{\partial J}{\partial d_{j}}=2\left[\sum_{r=0}^{n} d_{r} \int_{0}^{1} \gamma_{r}(t) \gamma_{j}(t) d t-\int_{0}^{1} f(t) \gamma_{j}(t) d t\right]=0 .
$$

Thus, we have

$$
\sum_{r=m}^{n} d_{r} \int_{0}^{1} \gamma_{r}(t) \gamma_{j}(t) d t=\int_{0}^{1} f(t) \gamma_{j}(t) d t-\beta_{j}
$$

where

$$
\beta_{j}=\sum_{r=0}^{m-1} d_{r} \int_{0}^{1} \gamma_{r}(t) \gamma_{j}(t) d t
$$

for each $j=m, m+1, \ldots, n$.

In order to find $y_{n}(t)$, we have to solve $(n-m)$ a system of linear equations while assuming $(n-m)$ unknowns $d_{r}$. The system (3.5) can be written in the form:

$$
G d=F
$$

where

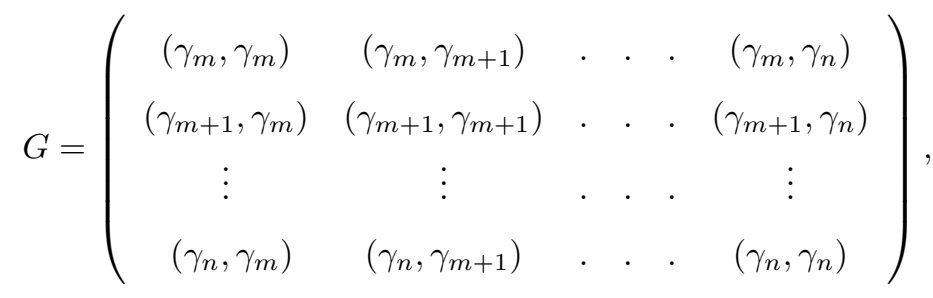

$$
\begin{aligned}
& d=\left[d_{m}, d_{m+1}, \ldots, d_{n}\right]^{T},
\end{aligned}
$$

and

$$
F=\left[\left(\gamma_{m}, f\right)-\beta_{m},\left(\gamma_{m+1}, f\right)-\beta_{m+1}, \ldots,\left(\gamma_{n}, f\right)-\beta_{n}\right]^{T}
$$

Definition 3.3. If Eq. (3.7) has a unique solution $d$, then $y_{n}(t)=\sum_{r=1}^{n} \frac{d_{r}}{r !} t^{r}$ is called an optimal squared approximation solution of Eqs. (1.1)-(1.2) defined on a set as $\operatorname{span}\left\{1, t, t^{2}, \ldots t^{n}\right\}, t \in[0,1]$.

Remark 3.4. If $\lim _{n \rightarrow \infty} \int_{0}^{1} T^{2}\left(t, y_{n}(t)\right) d t=0$, then the optimal squared approximation solution $y_{n}(t)$ converges to the exact solution $y(t)$ of Eqs. (1.1) and (1.2).

We are interested to know that as $n \rightarrow \infty$ the optimal squared approximation solution $y_{n}(t)$ will converge to the exact solution $y(t)$ of Eqs. (1.1) and (1.2). This conception is proven in Theorem 3.5. 
Theorem 3.5. Suppose $y(t), t \in[0,1]$ is an exact solution and $y_{n}(t)$ is an optimal squared approximation solution of Eqs. (1.1) and (1.2). If $\exists p_{n}(t)=\sum_{r=1}^{n} d_{r} t^{r}$ such that $\forall t \in[0,1], \lim _{n \rightarrow \infty} p_{n}(t)=y(t)$ then

$$
\lim _{n \rightarrow \infty} \int_{0}^{1} T^{2}\left(t, y_{n}(t)\right) d t=0
$$

Proof. The proof is similar to proof of Theorem 3 in [19].

\section{Illustrative EXAmples}

In this section, we use the presented method in Section 3 for solving two examples.

Example 4.1. For first example, consider the fractional integro-differential equation

$$
\begin{gathered}
D^{0.75} y(t)+\frac{1}{5} t^{2} e^{t} y(t)-\int_{0}^{t} x e^{t} y(x) d x=\frac{6 t^{2.25}}{\Gamma(3.25)}, \\
y(0)=0,
\end{gathered}
$$

where the exact solution is given by $y(t)=t^{3}$.

We applied the presented method with $n=3$ for solving this example and achieved the corresponding absolute errors in Table 1.

Table 1: Absolute errors for Example 1 for $n=3$.

\begin{tabular}{llllll}
\hline$t=0$ & $t=0.2$ & $t=0.4$ & $t=0.6$ & $t=0.8$ & $t=1.0$ \\
\hline 0 & $1.314 \times 10^{-15}$ & $1.045 \times 10^{-15}$ & $1.914 \times 10^{-16}$ & $2.475 \times 10^{-16}$ & $6.717 \times 10^{-16}$ \\
\hline
\end{tabular}

Example 4.2. Consider the equation

$$
\begin{gathered}
D^{\alpha} y(t)+\frac{7 t^{2}}{12} y(t)-\int_{0}^{1} t x y(x) d x-\int_{0}^{t}(x+t) y(x) d x=\frac{2 t^{2-\alpha}}{\Gamma(3-\alpha)}-\frac{t}{4}, \\
y(0)=0
\end{gathered}
$$

with the exact solution $y(t)=t^{2}$. By the presented method in section 3 for $n=2$ and different values of $\alpha$ absolute errors are reported in Table 2.

Example 4.3. Consider the equation $[20]$

$$
\begin{gathered}
D^{\alpha} y(t)+y(t)=t^{4}-\frac{1}{2} t^{3}-\frac{3}{\Gamma(4-\alpha)} t^{3-\alpha}+\frac{24}{\Gamma(5-\alpha)} t^{4-\alpha} \\
y(0)=0, \quad 0 \leq \alpha \leq 1
\end{gathered}
$$

whose exact solution is given by $y(t)=t^{4}-\frac{1}{2} t^{3}$. 
Table 2: Absolute errors for Example 2.

\begin{tabular}{llllll}
\hline $\mathrm{t}$ & $\alpha=0.2$ & $\alpha=0.5$ & $\alpha=0.7$ & $\alpha=0.9$ & $\alpha=1.0$ \\
\hline 0.0 & 0 & 0 & 0 & 0 & 0 \\
0.1 & 0 & $1.734 \times 10^{-17}$ & $1.387 \times 10^{-17}$ & 0 & 0 \\
0.2 & 0 & $3.469 \times 10^{-17}$ & $2.775 \times 10^{-17}$ & 0 & 0 \\
0.3 & 0 & $4.163 \times 10^{-17}$ & $4.163 \times 10^{-17}$ & 0 & 0 \\
0.4 & 0 & $5.551 \times 10^{-17}$ & $5.551 \times 10^{-17}$ & 0 & 0 \\
0.5 & 0 & $5.551 \times 10^{-17}$ & $5.551 \times 10^{-17}$ & 0 & 0 \\
0.6 & 0 & $5.551 \times 10^{-17}$ & $5.551 \times 10^{-17}$ & 0 & 0 \\
0.7 & 0 & $5.551 \times 10^{-17}$ & $1.110 \times 10^{-16}$ & 0 & 0 \\
0.8 & 0 & 0 & $1.110 \times 10^{-16}$ & 0 & 0 \\
0.9 & 0 & $1.110 \times 10^{-16}$ & $1.110 \times 10^{-16}$ & 0 & 0 \\
1.0 & 0 & 0 & $2.220 \times 10^{-16}$ & 0 & 0 \\
\hline
\end{tabular}

By taking different values of $\alpha$, we solved the above problem by means of the presented method. The maximum absolute error with the presented method and SCT method [20] for $n=4$ are compared in Table 3.

Table 3: Comparison of maximum absolute error for example 3.

\begin{tabular}{|c|c|c|c|}
\hline$\alpha$ & $\mathrm{n}=4$ & Present method & Method of [20] \\
\hline 0.01 & & $1.26 \times 10^{-14}$ & $1.2 \times 10^{-5}$ \\
\hline 0.1 & & $1.41 \times 10^{-14}$ & $1.3 \times 10^{-4}$ \\
\hline 0.5 & & $5.30 \times 10^{-15}$ & $7.8 \times 10^{-4}$ \\
\hline 0.99 & & $1.30 \times 10^{-15}$ & $8.6 \times 10^{-4}$ \\
\hline 1 & & $1.14 \times 10^{-15}$ & $8.6 \times 10^{-4}$ \\
\hline
\end{tabular}

Example 4.4. Consider the equation

$$
\begin{gathered}
D^{\frac{3}{2}} y(t)+y(t)=\frac{6}{\Gamma(2.5)} t^{1.5}+\frac{6}{\Gamma(1.5)} t^{0.5}+t^{3}+t^{2} \\
y(0)=0, y^{\prime}(t)=0
\end{gathered}
$$

whose exact solution is given by $y(t)=t^{3}+t^{2}$.

By applying the technique described in section 3 with $\mathrm{m}=4$, we approximate solution as

$$
y(t)=\sum_{r=0}^{4} \frac{y^{(r)}(0)}{r !} t^{r}=\sum_{r=0}^{4} \frac{d_{r}}{r !} t^{r}
$$


Here, by using Eq. (3.7), we obtain

$$
\left(\begin{array}{ccc}
1.00901 & 0.422206 & 0.123762 \\
0.422206 & 0.19103 & 0.0586763 \\
0.123762 & 0.0586763 & 0.0186265
\end{array}\right)\left(\begin{array}{l}
d_{2} \\
d_{3} \\
d_{4}
\end{array}\right)=\left(\begin{array}{c}
4.55127 \\
1.9906 \\
0.599582
\end{array}\right)
$$

Finally by solving Eq.(4.14), we get

$$
d_{2}=2, d_{3}=6, d_{4}=0
$$

Thus we can write

$$
y(t)=d_{0}+d_{1} t+d_{2} \frac{t^{2}}{2 !}+d_{3} \frac{t^{3}}{3 !}+d_{4} \frac{t^{4}}{4 !}=t^{3}+t^{2}
$$

which is the exact solution.

\section{CONClusion}

In this paper, we proposed least squares approximation method to solve a class of linear fractional integro-differential equations comprising of Fredholm and Volterra cases based on a polynomial of degree $n$. The numerical experiments show that the proposed method can be suitable method for solving these equations.

\section{REFERENCES}

[1] C. A. Monje, Y. Chen, B. M. Vinagre, D. Xue, and V. Feliu, Fractional-Order Systems and Controls, Advances in Industrial Control, Springer, London, UK, 2010.

[2] S. Das, Functional Fractional Calculus for System Identification and Controls, Springer, New York, 2008.

[3] I. Podlubny, Fractional Differential Equations, Academic Press Inc., San Diego, CA, 1999.

[4] R.L. Magin, Fractional Calculus in Bioengineering, Begell House Publishers, 2006.

[5] M. Amairi, M. Aoun, S. Najar, M.N. Abdelkrim, A constant enclosure method for validating existence and uniqueness of the solution of an initial value problem for a fractional differential equation, Appl. Math. Comput. 217 (2010), 2162-2168.

[6] K. Diethelm, N.J. Ford, Multi-order fractional differential equations and their numerical solutions, Appl. Math. Comput. 154 (2004), 621-640.

[7] J. Deng, L. Ma, Existence and uniqueness of solutions of initial value problems for nonlinear fractional differential equations, Appl. Math. Lett. 23 (2010), 676-680.

[8] A.A. Kilbas, H.M. Srivastava, J.J. Trujillo, Theory and Applications of Fractional Differential Equations, Elsevier, San Diego, 2006.

[9] Kumar P, Agrawal OP, Numerical scheme for the solution of fractional differential equations of order greater than one, J. Comput. Nonlinear Dynam. 1 (2006), 178-185.

[10] S.A.EI-Wakil, A. Elhanbaly, M. A. Abdou, Adomian decomposition method for solving fractional nonlinear differential equations, Appl. Math. Comput. 182 (2006), 313-324.

[11] Momani S, Odibat Z, Numerical approach to differential equations of fractional order, J. Comput. Appl. Math. 207 (2007), 96-110. 
[12] Jabari, D. Ezzati, R. and Maleknejad, K. A new operational matrix for solving two-dimensional nonlinear integral equations of fractional order. Cogent Math. 4 (2017), Art. ID 1347017.

[13] Li Y, Zhao W, Haar wavelet operational matrix of fractional order integration and its applications in solving the fractional order differential equations, Appl. Math. Comput. 216 (2010), 2276-2285.

[14] Rawashdeh EA, Numerical solution of fractional integro-differential equations by collocation method, Appl. Math. Comput. 176 (2006), 1-6.

[15] Atanackovic TM, Stankovic B, On a numerical scheme for solving differential equations of fractional order, Mech. Res. Commun. 35 (2008), 429-438.

[16] Pedas Arvet, Tamme Enn, Spline collocation methods for linear multi-term fractional differential equations, J. Comput. Appl. Math. 236 (2011), 167-176.

[17] Odibat Z, Momani S, Numerical methods for nonlinear partial differential equations of fractional order, Appl. Math. Model. 32 (2008), 28-39.

[18] Wu JL, A Wavelet operational method for solving fractional partial differential equations numerically, Appl Math. Comput. 214 (2009), 31-40.

[19] Q. Wang, K. Wang, S. Chen, Least squares approximation method for the solution of Volterra-Fredholm integral equations, J. Comput. Appl. Math. 272 (2014), 141-147.

[20] A.H. Bhrawy, M.M. Tharwat, A. Yildirim Duan, A new formula for fractional integrals of Chebyshev polynomials: Application for solving multi-term fractional differential equations, Appl. Math. Model. 37 (2013), 4245-4252. 